\title{
IL-23 links glutamine addiction and immune function
}

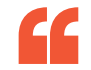

the study

reveals a

previously

uncharacter-

ized immuno-

regulatory role

for IL-23 in

glutamine-

addicted

cCRCC

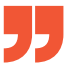

A key metabolic hallmark of clear cell renal cell carcinoma (ccRCC) is glutamine addiction, which depletes the local microenvironment of this metabolite. However, the influence of tumour glutamine metabolism on the ccRCC immune contexture is unclear. A new study now offers mechanistic insight into how macrophage-derived interleukin-23 (IL-23) orchestrates immune infiltration in glutamine-addicted ccRCC tumours.

Using The Cancer Genome Atlas (TCGA) data and tumour samples from patients, $\mathrm{Fu}$ and colleagues initially found that a 41-gene glutamine metabolism signature - indicative of tumour glutamine metabolism - was associated with poor overall survival (OS) and cancer-specific survival (CSS), decreased tumour-infiltrating $\mathrm{CD}^{+}$ $\mathrm{T}$ cell cytotoxicity, and increased regulatory $\mathrm{T}\left(\mathrm{T}_{\text {reg }}\right)$ cell-associated gene expression in ccRCC tumours. Interestingly, IL-23 expression was positively correlated with both glutamine metabolism and $\mathrm{T}_{\text {reg }}$ cell signatures and was associated with poor OS and CSS. Collectively, these data suggested that glutamine addiction dampens $\mathrm{T}$ cell-mediated antitumour cytotoxicity via $\mathrm{T}_{\text {reg }}$ cell

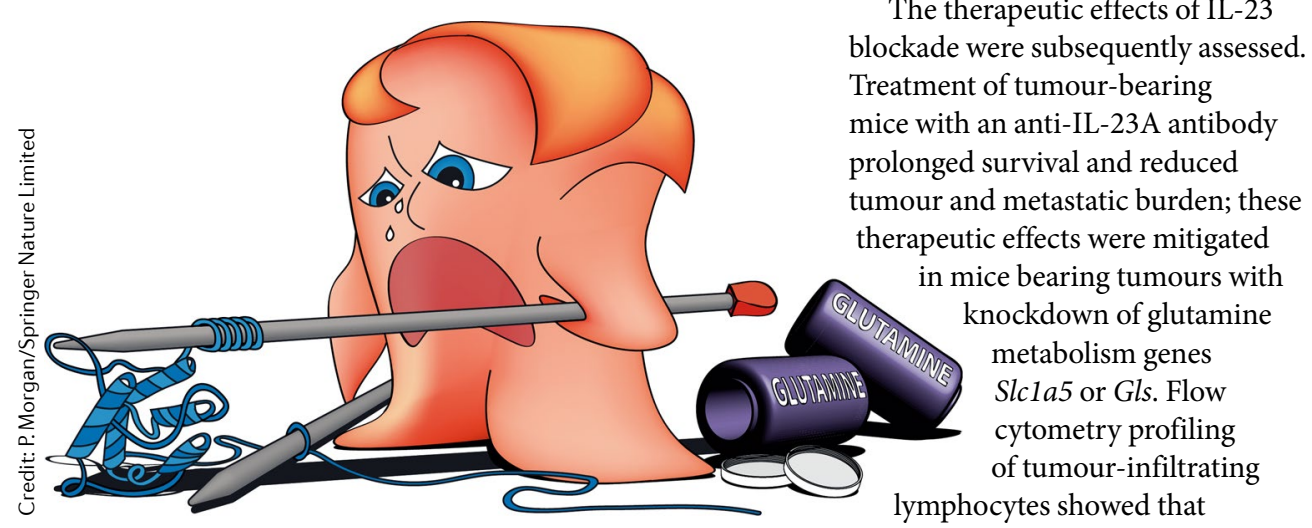

activation and implicated IL-23 as the missing link between glutamine metabolism and $\mathrm{T}_{\text {reg }}$ cell responses.

The role of IL-23 in regulating $\mathrm{T}_{\text {reg }}$ cell function was then investigated. In co-culture experiments, recombinant IL-23 treatment increased $\mathrm{T}_{\text {reg }}$ cell proliferation and activation, elevated $\mathrm{T}_{\text {reg }}$ cell-derived transforming growth factor- $\beta$ (TGF $\beta$ ) and IL-10 expression, and enhanced $\mathrm{T}_{\text {reg }}$ cell-dependent suppression of responder $\mathrm{CD}^{+}$and $\mathrm{CD} 8^{+} \mathrm{T}$ cell proliferation, inferring that IL-23 enhances $\mathrm{T}_{\text {reg }}$ cell immunosuppressive functionality. Flow cytometry and immunofluorescence assays identified macrophages as the predominant source of IL-23 in human ccRCC tumours. Furthermore, culture of murine peritoneal macrophages and isolated human tumour-infiltrating macrophages under glutaminedeficient conditions increased IL-23 secretion, suggesting that glutamine deprivation in the glutamine-addicted ccRCC tumour microenvironment promotes IL-23 secretion. Subsequent experiments identified a role for hypoxiainducible factor $1 \alpha$ (HIF1 $\alpha$ ) in IL-23 induction in glutamine-deprived macrophages.

The therapeutic effects of IL-23 blockade were subsequently assessed. Treatment of tumour-bearing prolonged survival and reduced tumour and metastatic burden; these in mice bearing tumours with ockdown of glutamine metabolism genes Slc1a 5 or Gls. Flow cytometry profiling lymphocytes showed that
anti-IL-23A therapy decreased $\mathrm{T}_{\text {reg }}$ cell infiltration and increased activated $\mathrm{CD} 8^{+} \mathrm{T}$ cell counts, indicative of enhanced antitumour cytotoxicity. Furthermore, anti-IL23A antibody therapy synergistically enhanced the antitumour effect of an anti-programmed cell death 1 (PD-1) antibody, highlighting the potential for IL-23 blockade to improve the efficacy of immune checkpoint inhibition in ccRCC.

Fu et al. also developed a novel in vitro culture system incorporating bulk single-cell suspensions from resected ccRCC tumours to simulate the in vivo tumour immune contexture. "This model is easy to handle and can maintain the proportions and phenotype of leukocyte subsets during short-time culture," explains author Jiejie $\mathrm{Xu}$. In this system, treatment with the FDA-approved anti-IL-23A antibody guselkumab decreased $\mathrm{T}_{\text {reg }}$ cell proliferation, increased $\mathrm{CD}^{+} \mathrm{T}$ cell cytotoxicity and induced tumour cell death.

Overall, the study reveals a previously uncharacterized immunoregulatory role for IL-23 in glutamine-addicted ccRCC tumours and highlights therapeutic opportunities for IL-23 blockade in this context, either alone or in combination with immune checkpoint inhibitors. "Further investigation of the impact of IL-23 on other immune or stromal cells is still needed to improve our understanding of the biological functions of IL-23," concludes Xu.

Conor A. Bradley

ORIGINAL ARTICLE Fu, Q. et al. Tumorassociated macrophage-derived interleukin-23 interlinks kidney cancer glutamine addiction with immune evasion. Eur. Urol. https://doi.org/ 10.1016/j.eururo.2018.09.030(2018) 